\title{
Near and sub-barrier reactions of ${ }^{8} \mathrm{~B}$
}

\author{
J. J. Kolata ${ }^{1, \star}$, Eli F. Aguilera ${ }^{2, \star \star}$, and Valdir Guimarães ${ }^{3, \star \star \star}$ \\ ${ }^{1}$ Physics Department, University of Notre Dame, Notre Dame, Indiana, 46556-5670, USA \\ ${ }^{2}$ Departamento de Aceleradores, Instituto Nacional de Investigaciones Nucleares, Apartado Postal 18-1027, Código Postal \\ 11801, México, Distrito Federal, México \\ ${ }^{3}$ Instituto de Fisica, Universidade de São Paulo, Rua do Matão Nr. 1371, 05508-900, São Paulo, SP, Brazil
}

\begin{abstract}
Four classes of measurements that are important for achieving a complete understanding of the reactions of exotic nuclei are identified and classified according to their degree of difficulty. Previously reported data for near- and sub-barrier fusion of ${ }^{8} \mathrm{~B}+{ }^{58} \mathrm{Ni}$ are critically reviewed. The influence of breakup protons on the evaporation proton measurements for this system is shown to be small at all energies except for the lowest one measured, and corrections are made for this process. Model dependencies in the deduced fusion cross sections are assessed using three different evaporation codes. Data sets for targets of ${ }^{58} \mathrm{Ni}$ and ${ }^{28} \mathrm{Si}$ are shown to be consistent with each other, and with fusion enhancement up to energies that are greater than the Coulomb barrier $\mathrm{V}_{b}\left(\mathrm{E}_{c . m .} . \lesssim \mathrm{V}_{b}+1.5 \times \hbar \omega\right)$. An important difference with the behavior of neutron-halo systems is thereby confirmed. Possible fusion suppression beyond this energy is suggested by the ${ }^{28} \mathrm{Si}$ data. A proposal to measure the ${ }^{8} \mathrm{~B}+{ }^{40} \mathrm{Ar}$ fusion cross section using an active-target time projection chamber is discussed.
\end{abstract}

\section{Introduction}

The study of nuclear reactions of exotic, weakly-bound nuclei near the limits of stability has revealed a large number of interesting features. However, most of these studies have been carried out at energies far above the Coulomb barrier, due to the relative unavailability of these beams at Coulomb-barrier energies. As a result there is only a rather limited literature on reactions, such as complete and incomplete fusion, which turn out to be very important for achieving a complete understanding of the exotic structure of these nuclei (see Ref. [1] and references therein). Four different types of relevant experiments can be identified and ranked according to their relative degree of difficulty. The first of these is elastic scattering, the easiest reaction to study because of its large cross section, which leads to a determination of the total reaction cross section. As discussed below, this can be an important indicator of the effect of structure on nuclear reactions, and in some cases the effects of coupling to weakly-bound direct channels on elastic scattering can be dramatic, as illustrated in Fig. 1.

The second category of experiments, measurement of transfer/breakup reactions, is also relatively easy but complicated by the fact that separation of various reaction types such as one- and two-particle transfer from direct breakup can be challenging. This separation was accomplished for the ${ }^{6} \mathrm{He}+{ }^{209} \mathrm{Bi}$ reaction in a series of experiments [3-5] carried out at the TwinSol facility [6]. Other examples are discussed in Ref. [1].

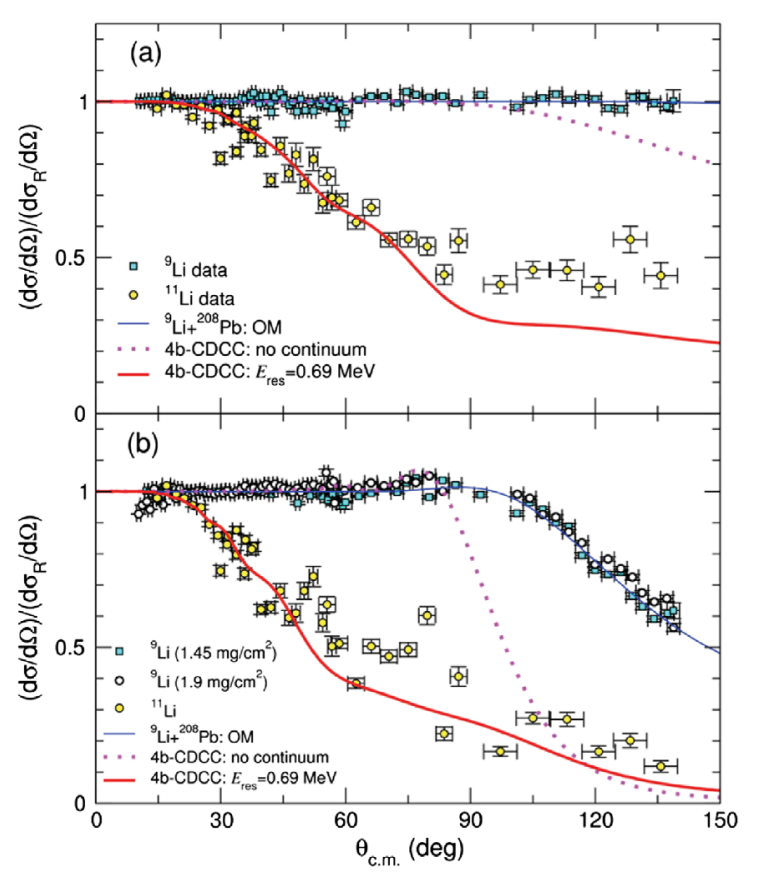

Figure 1. Elastic-scattering cross section (ratio-to-Rutherford) for ${ }^{9} \mathrm{Li}$ and ${ }^{11} \mathrm{Li}$ on a ${ }^{208} \mathrm{~Pb}$ target at the same c.m. energies of $23.1 \mathrm{MeV}$ (upper panel) and $28.3 \mathrm{MeV}$ (lower panel). Figure reprinted with permission from Ref. [2]. (C)2012, The American Physical Society.

\footnotetext{
^e-mail: jkolata@nd.edu

$\star \star$ e-mail: eli.aguilera@inin.gob.mx

$\star \star \star$ e-mail: valdirg@if.usp.br
} 
The third category, most relevant for this conference, is fusion. Although fusion cross sections tend to be quite large above the Coulomb barrier, they rapidly grow smaller below the barrier where the most interesting effects are expected. Experimental techniques range from direct detection of the fusion products, to the light particles evaporated from them, to $\gamma$ radiation emitted by the final systems. In the case of weakly-bound projectiles, it can be very difficult to separate complete fusion from incomplete fusion, i.e., the fusion of a part of the projectile with the target. As a result, only a few examples of the separation of these two types of fusion reactions, the fourth category of measurement, have been reported for exotic nuclei, and these are typically at above-barrier energies. Examples include Refs. [7, 8]. Another issue addressed by fusion studies of radioactive but not weakly-bound projectiles is the influence of neutron number on the fusion cross section, as for example in Refs. $[9,10]$ and a work reported at this conference [11].

\section{Reactions of ${ }^{8} \mathrm{~B}$}

\subsection{Elastic scattering and breakup}

The elastic scattering of ${ }^{8} \mathrm{~B}$ on ${ }^{58} \mathrm{Ni}$ has been measured [12], and the extracted total reaction cross section was compared with that for other weakly- and strongly-bound projectiles on various targets in Ref. [13]. The reduced total reaction cross sections are illustrated in Fig. 2.

It can be seen in this figure that the weakly-bound, two-neutron-halo nucleus ${ }^{6} \mathrm{He}$ and the one-proton-halo nucleus ${ }^{8} \mathrm{~B}$ both display approximately the same reduced total reaction cross sections. This is surprising since it is

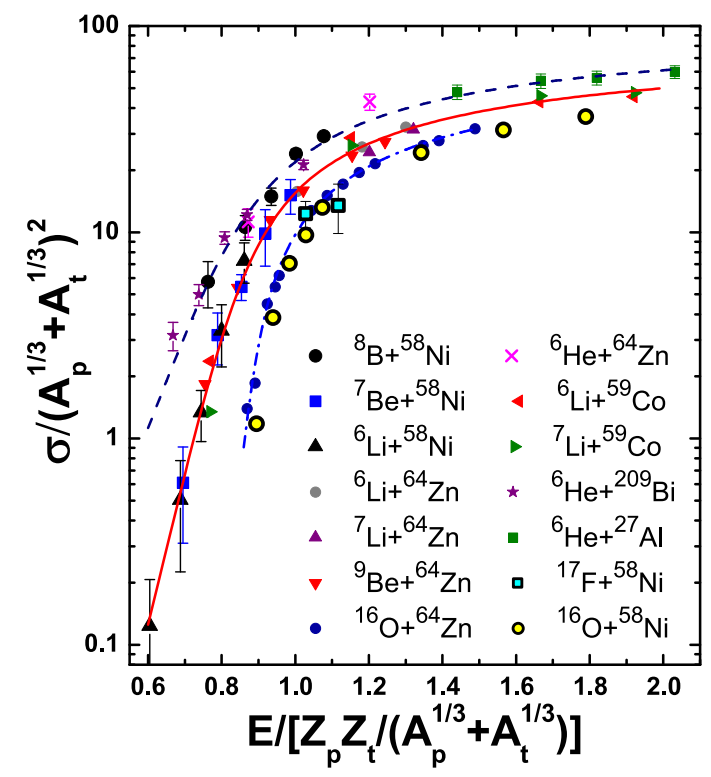

Figure 2. Reduced total reaction cross sections for a number of weakly- and strongly-bound projectiles (from Ref. [13]). (C)2009, The American Physical Society. known that the two-neutron-transfer cross section for ${ }^{6} \mathrm{He}$ is remarkably large near to and below the Coulomb barrier [3], while the breakup cross section of ${ }^{8} \mathrm{~B}$ is considerably smaller $[14,15]$. The latter was extensively studied in the context of the coupled discretized continuum channels (CDCC) method [16]. The agreement with the data was excellent, so transfer/breakup is well understood for this system. It appeared, therefore, that the fusion cross section for ${ }^{8} \mathrm{~B}$ might show some surprises.

\subsection{Fusion}

The fusion cross section for ${ }^{8} \mathrm{~B}+{ }^{58} \mathrm{Ni}$ was measured [17] by detecting evaporation protons emitted at backward angles. This method has an inherent advantage since the ${ }^{66}$ As compound system is at the proton dripline, resulting in the emission of about 2.3 protons per fusion event and thereby increasing the statistical accuracy of the deduced cross section. However, it was suggested that the evaporation proton yield might have been contaminated by direct breakup protons even at backward angles [18]. Therefore, an intensive study of this possibility was carried out [19]. The calculations of Ref. [16] were extended to predict the direct proton yield at backward angles. It was found that the contamination was very small except at the lowest energy measured, and appropriate corrections were deduced. Furthermore, the evaporation $\alpha$-particle yield was shown to agree with the proton yield. The statistics were much poorer since the $\alpha$-particle multiplicity is only about 0.5 . However, it was possible to recover the fusion cross section at the lowest energy since the $\alpha$-particle yield is not expected to be contaminated by direct processes. In addition, possible systematic errors due to the calculation of the evaporated-particle multiplicities were determined. The same methodology was applied to the ${ }^{8} \mathrm{~B}+{ }^{28} \mathrm{Si}$ data [20] and the results are shown in Fig. 3.

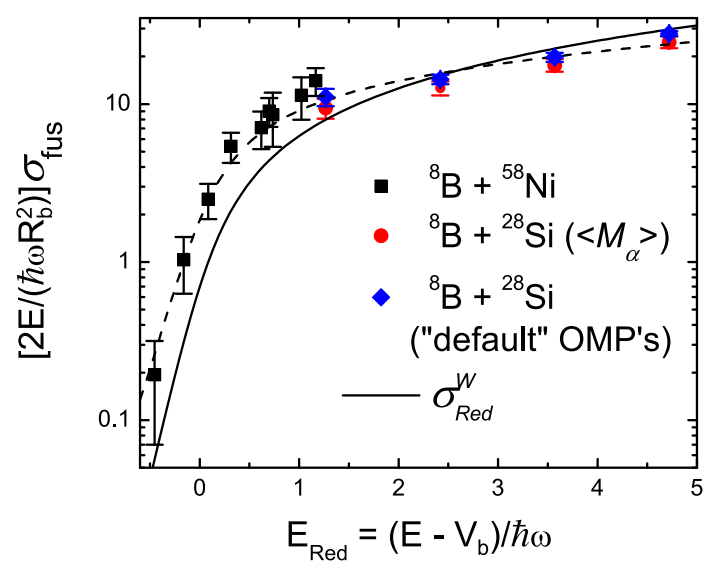

Figure 3. Reduced total fusion yields for ${ }^{8} \mathrm{~B}+\left({ }^{58} \mathrm{Ni},{ }^{28} \mathrm{Si}\right.$ ) (from Ref. [13]). The solid curve represents the Universal Fusion Function (UFF) [21]. (c)2016, The American Physical Society. 
The solid curve in this figure is the Universal Fusion Function (UFF) [21], which represents the expectation for fusion in "normal" circumstances. It can be seen that ${ }^{8} \mathrm{~B}+{ }^{58} \mathrm{Ni}$ fusion appears to be enhanced up to about 1.5 times the reduced barrier, while the ${ }^{8} \mathrm{~B}+{ }^{28} \mathrm{Si}$ fusion cross section indicates a possible fusion suppression at energies well above the barrier. The ${ }^{58} \mathrm{Ni}$ and ${ }^{28} \mathrm{Si}$ data agree reasonably well with each other at the one common (reduced) energy point. (It should be noted, however, that the ${ }^{28} \mathrm{Si}$ cross sections shown are the result of a reanalysis taking into account the fact that different evaporation codes predict different $\alpha$-particle multiplicities. See Ref. [19] for a further discussion). In addition, the sum of the ${ }^{58} \mathrm{Ni}$ total fusion cross section plus the direct breakup yield equals the experimentally-measured total reaction cross section (Fig. 4). This is similar to the situation for ${ }^{6} \mathrm{He}$ reactions, in which the sum of the fusion and breakup/transfer yields equals the total reaction cross section. However, in the ${ }^{6} \mathrm{He}$ case, direct reactions, and particularly two-neutron transfer [3], dominate the total reaction cross section near the barrier, while in the case of ${ }^{8} \mathrm{~B}$ the fusion and direct yields are more comparable.

Nevertheless, there are two important points to consider here. First of all, the cross sections shown in Fig. 3 depend on light-particle multiplicities calculated by various evaporation-model codes which have been shown to disagree with each other (though more-so for $\alpha$-particle than proton evaporation). It would be helpful to compare with data from direct detection of evaporation residues, but this is a very difficult experiment at the available ${ }^{8} \mathrm{~B}$ beam intensities. Secondly, it is very likely that the total fusion yield shown in this figure includes a large component of incomplete fusion (ICF) in which the ${ }^{7} \mathrm{Be}$ core fuses with the target while the weakly-bound proton continues on relatively undisturbed. The $\gamma$-ray detection methods that can resolve complete fusion (CF) from ICF processes [8] also require much greater beam intensities than are currently

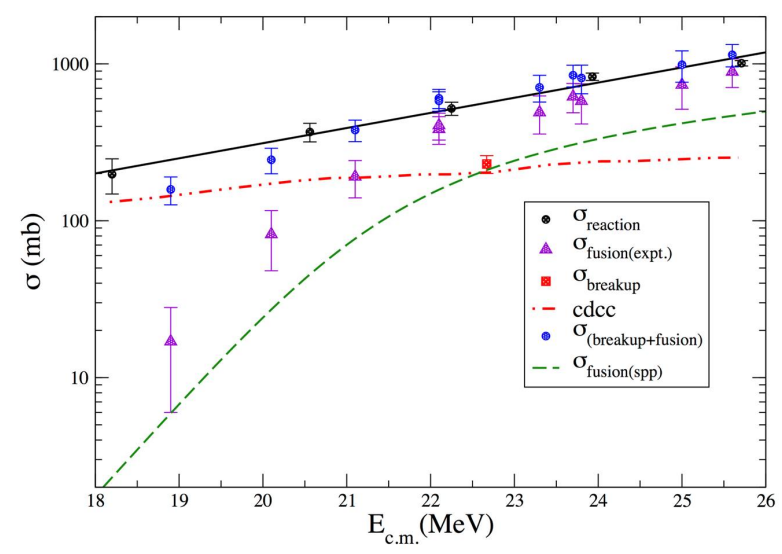

Figure 4. Cross sections for ${ }^{8} \mathrm{~B}+{ }^{58} \mathrm{Ni}$. The total reaction cross section is from Refs. $[12,13]$, the total fusion cross section from Refs. [17, 19], and the breakup cross section from Refs. [14, 15] and also the CDCC calculation [16]. The fusion curve was calculated using the Sào Paulo potential [22]. available. In the next section, we will discuss an experimental technique that can address both these issues.

\section{Fusion Studies with a Time Projection Chamber}

In a recent experiment [23] using the prototype activetarget time projection chamber (pAT-TPC) [24] developed at Michigan State University, the fusion cross section for ${ }^{10} \mathrm{Be}+{ }^{40} \mathrm{Ar}$ was studied with extremely high efficiency. An entire excitation function (Fig. 5) was measured with an average beam intensity of only 100 particles per second in a counting period of 90 hours.

Furthermore, it was possible to directly image the evaporated charged particles (protons and $\alpha$-particles), so that events in which only neutrons were emitted (NCP fusion) could be separated from the charged-particle (CP) events (Fig. 6). The individual cross sections for these types of events, as well as the summed fusion yield, agreed well with the predictions of the PACE4 fusion-evaporation code contained within the LISE++ package [25], as shown in Fig. 5. Note, however, that the target was actually P10 gas $\left(90 \%\right.$ Ar plus $\left.10 \% \mathrm{CH}_{4}\right)$ so that the $\mathrm{C}$ contaminant needed to be dealt with. As a result, the ${ }^{40} \mathrm{Ar}$ fusion yield at low energies was not well determined. See Ref. [23] for further details.

The results illustrated in Figs. 5 and 6 suggest that it will be possible to determine the ${ }^{8} \mathrm{~B}+{ }^{40} \mathrm{Ar}$ fusion yield by directly imaging the evaporation products, thus eliminating ambiguities due to the reliance on multiplicity calculations in the experiments discussed above. Furthermore, the ability to image light charged particles in this experiment suggests that it might be possible to distinguish complete fusion from incomplete fusion by looking for the associated higher-energy proton from ${ }^{8} \mathrm{~B}$ breakup, as well as by measuring the kinetic energy of the recoiling evaporation residue. A calculation of the expected results for complete

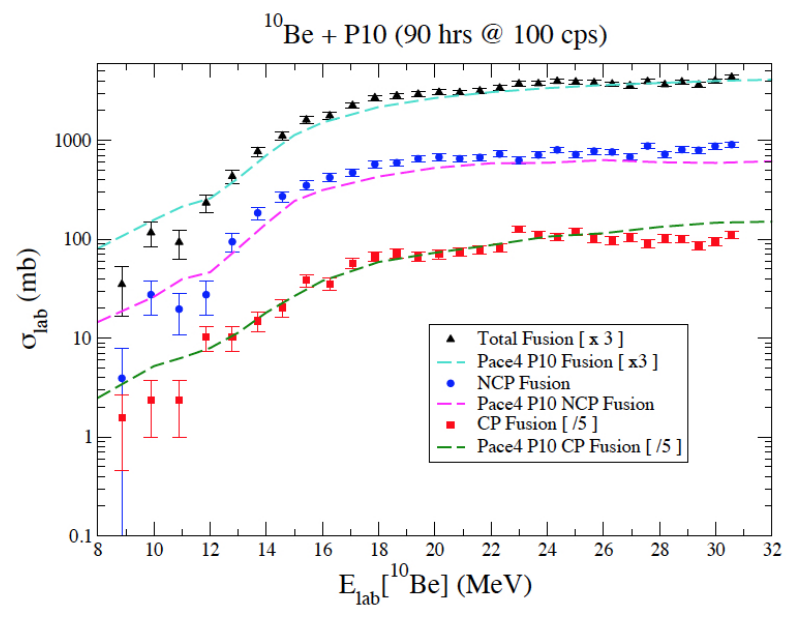

Figure 5. Total fusion cross section for ${ }^{10} \mathrm{Be}+\mathrm{P} 10$ (from Ref. [23]). The individual yields for neutron-only evaporation (NCP events) and charged-particle evaporation (CP events) are also shown. The curves are PACE4 calculations. (C)2016, Elsevier. 
fusion is shown in Fig. 7 below. The cross sections were calculated using PACE4. The red points and associated curve are for a target of P10.

The blue points and curve assume a target of $90 \% \mathrm{Ar}$ plus $10 \% \mathrm{H}_{2}$. This mixture worked well in a test chamber, but problems with the pAT-TPC (since repaired) prevented its use in the previous experiment. Incomplete fusion, if present, should act to increase the illustrated yield but hopefully could be identified as discussed above. This proposed experiment would then be an excellent test of the ability to separate CF from ICF for very-low-intensity exotic beams.

\section{Modifications to TwinSol}

One very important impediment to the proposed ${ }^{8} \mathrm{~B}$ fusion studies with a TPC is the fact that this beam was heavily contaminated by ${ }^{6} \mathrm{Li}$ and ${ }^{7} \mathrm{Be}$ in the previous experiments at TwinSol, such that ${ }^{8} \mathrm{~B}$ was only a very minor component. This was not a problem since ${ }^{8} \mathrm{~B}$ could be unambiguously identified by its time of flight to the secondary target. However, TPC's have very definite rate limitations and therefore require a pure beam. As a result, extensive modifications of TwinSol are being carried out in order to provide such a beam. These are part of a major project to also provide higher-intensity and higher- $\mathrm{Z}$ radioactive beams.

The gas-cell primary target has been doubled in length to $5 \mathrm{~cm}$, and 4 micron Ti windows have been installed ( 3 micron $\mathrm{Ti}$ is currently being tested). In addition, a drive mechanism that allows four different gas cells to be used before the chamber must be opened to replace damaged windows has been developed. To accomplish this, the primary target was moved upstream from the first solenoid by an additional $7 \mathrm{~cm}$. Next, the fixed-aperture collimator at the crossover point between the two solenoids was

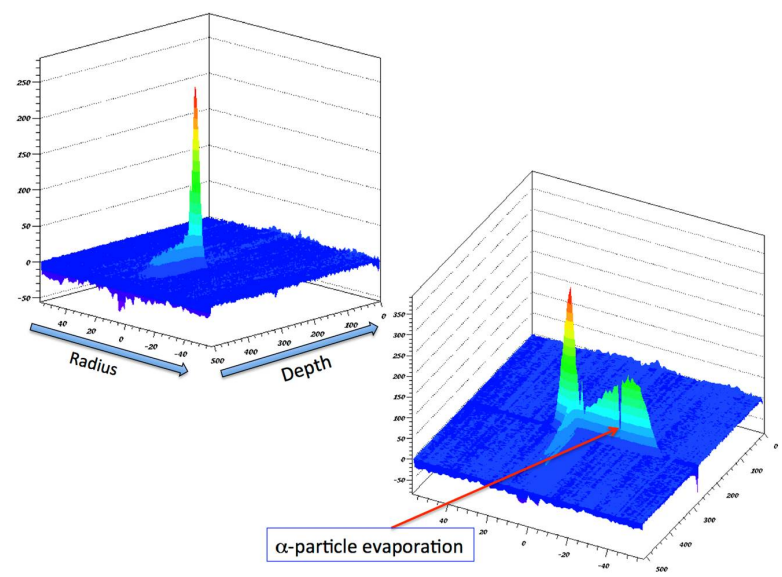

Figure 6. Ionization vs. radius and depth in the counter for two isolated fusion events. The leftmost plot shows a typical neutronevaporation (NCP) event. The rightmost plot shows the effect of $\alpha$-particle evaporation from the fused system. Note the deviation of the fusion-residue peak from the beam track, and its larger width, due to recoil of the evaporation product. (from Ref. [23]). (C)2016, Elsevier. replaced by a variable iris that can be adjusted without breaking vacuum. This collimator determines the momentum acceptance of the system and therefore can improve the purity of the transmitted beam. Finally, the focus of the secondary beam was moved about $5 \mathrm{~m}$ upstream. Due to space limitations, the pAT-TPC in previous experiments was installed at about $9 \mathrm{~m}$ from the primary target, while the designed focal point of TwinSol was at $5.6 \mathrm{~m}$. As a result, the size of the beam was increased and therefore the transmission into the TPC was reduced. By moving the second solenoid $88 \mathrm{~cm}$ upstream, the optics were substantially improved and it was possible to achieve a reasonable beam image at $11 \mathrm{~m}$. Another advantage of this is that the beam now travels through an aperture in a $1.25 \mathrm{~m}$ wide high-density concrete shielding wall, which greatly reduces the background levels for anticipated neutron and $\gamma$-ray experiments with radioactive beams.

The effects of all these modifications were investigated and optimized with Monte-Carlo calculations using LISE++ [25], which now includes calculation blocks for TwinSol. The ability to include angular distribution data

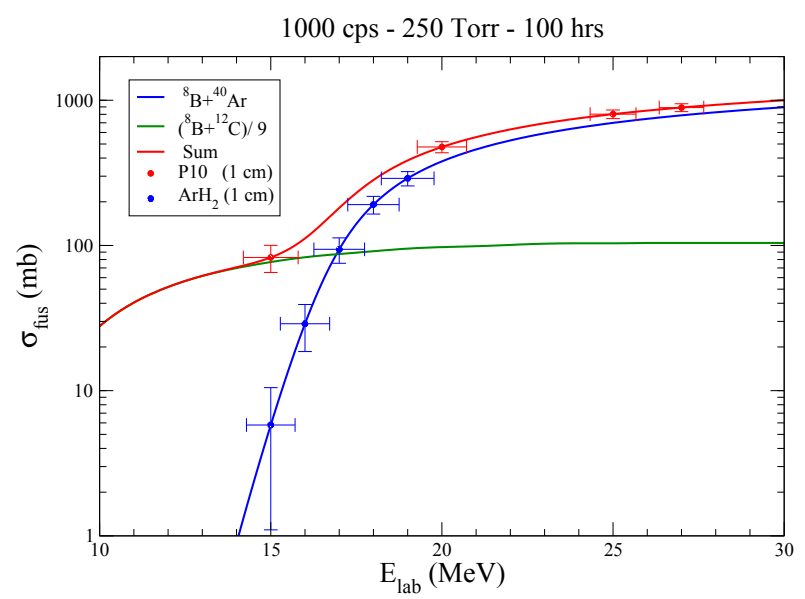

Figure 7. Calculated complete fusion cross sections for ${ }^{8} \mathrm{~B}+{ }^{40} \mathrm{Ar}$. The cross sections are averaged over a $1 \mathrm{~cm}$ path length of the incident ${ }^{8} \mathrm{~B}$, resulting in the horizontal and vertical error bars illustrated.

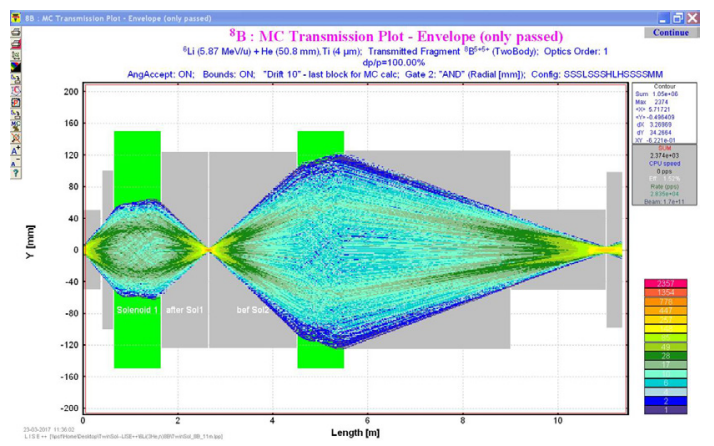

Figure 8. LISE++ calculation of the envelope of a $27-\mathrm{MeV}^{8} \mathrm{~B}$ beam focussed at $11 \mathrm{~m}$ from the primary target. 


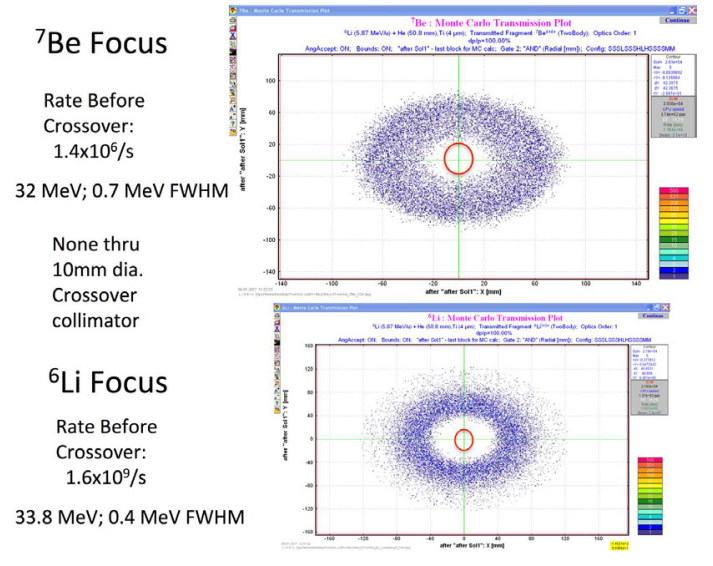

Figure 9. LISE++ calculation for the ${ }^{7} \mathrm{Be}$ and ${ }^{6} \mathrm{Li}$ images at the crossover collimator, including scattering in the gas-cell windows. The red circles illustrate $10 \mathrm{~mm}$ diameter collimators in each case. All the ${ }^{8} \mathrm{~B}$ ions pass through a collimator of this diameter, leading to a rate of $3.6 \times 10^{4} / \mathrm{s}$ through an $8 \mathrm{~mm}$ diameter collimator at the final focus (assuming a $1 \mathrm{e} \mu \mathrm{A}{ }^{6} \mathrm{Li}\left(3^{+}\right)$primary beam).

for the production reaction in this code (either from experimental data or DWBA calculations) turned out to be very helpful both in estimating the final secondary beam rate and in adjusting the solenoid currents to maximize that rate. A sample calculation for a $27-\mathrm{MeV}^{8} \mathrm{~B}$-ion envelope is illustrated in Fig. 8. The calculations suggest that the purity of the beam might be greater than $90 \%$ (see Fig. 9 below).

\section{Summary and Conclusions}

Elastic scattering, breakup, and fusion reactions of the exotic one-neutron-halo nucleus ${ }^{8} \mathrm{~B}$ have been studied over a period of several years. The recent fusion data suggests that the total fusion cross section of this projectile is enhanced near and below the Coulomb barrier, while apparently being suppressed at energies well above the barrier. The sum of total fusion plus breakup yields equals the measured large total reaction cross section. This is similar to the situation for ${ }^{6} \mathrm{He}$ except that, in that case, the total reaction cross section near the barrier is dominated by neutron transfer, while the fusion yield is more similar to the direct-reaction yield for ${ }^{8} \mathrm{~B}$.

It seems likely that incomplete fusion (ICF) plays a substantial role in enhancing the ${ }^{8} \mathrm{~B}$ total fusion cross section. A possible method to measure the ICF yield using a time projection chamber has been proposed. This method requires beams of high purity. Measures currently underway at TwinSol to improve the purity of the radioactive beams, as well as to provide higher- $Z$ beams and a more background-free area for use in $\gamma$-ray and neutron detection experiments, have been described. The ${ }^{8} \mathrm{~B}$ plus ${ }^{40} \mathrm{Ar}$ fusion experiment would be an excellent test of the ability of TPC's to detect and measure ICF reactions with very-low-intensity exotic beams.

\section{Acknowledgements}

This work has been partially supported by the US National Science Foundation under Grant No. PHY14-01343, and by the CONACYT (México). We also wish to thank Dr. Oleg Tarasov of Michigan State University for his help in using LISE++ and working to improve its TwinSol predictions.

\section{References}

[1] J.J. Kolata, V. Guimarães, and E. F. Aguilera, European Physical Journal A 52, 123 (2016).

[2] M. Cubero, et al., Phys. Rev. Lett. 109, 262701 (2012).

[3] J. P. Bychowski, et al., Phys. Lett. B 596, 26 (2004).

[4] P. A. DeYoung, et al., Phys. Rev. C 71, 051601(R) (2005).

[5] J.J. Kolata, et al., Phys. Rev. C 75, 031302[R] (2007).

[6] M. Y. Lee et al., Nucl. Instrum. Methods in Physics Research A 422, 536 (1999).

[7] S.A. Krupko, et al., AIP Conf. Proc. 1098, 245 (2009).

[8] D.J. Hinde and M. Dasgupta, Phys. Rev. C 81, 064611 (2010).

[9] J.J. Kolata, et al., Phys. Rev. C 85, 054603 (2012).

[10] P.F.F. Carnelli, et al., Phys. Rev. Lett. 112, 192701 (2014).

[11] R.T. deSouza, et al., Phys. Rev. C 88, 014602 (2013).

[12] E. F. Aguilera, et al., Phys. Rev. C 79, 021601[R] (2009).

[13] J.J. Kolata, et al., Phys. Rev. C 79, 027603 (2009).

[14] J.J. Kolata, et al., Phys. Rev. C 63, 024616 (2001).

[15] E. F. Aguilera, et al., Physics of Atomic Nuclei 71, 1163 (2008).

[16] J.A. Tostevin, et al., Phys. Rev. C 63, 024617 (2001).

[17] E. F. Aguilera, et al., Phys. Rev. Lett. 107, 092701 (2011).

[18] J. Rangel, et al., Eur. Phys. J. A 49, 57 (2013).

[19] E. F. Aguilera, P. Amador-Valenzuela, et al., Phys. Rev. C 93, 034613 (2016).

[20] A. Pakou, et al., Phys. Rev. C 87, 014619 (2013).

[21] L.F. Canto, et al., Nucl. Phys. A 821, 51 (2009).

[22] L.C. Chamon, et al., Phys. Rev. C 66, 014610 (2002).

[23] J.J. Kolata, et al., Nucl. Instrum. Methods in Physics Research A 830, 82 (2016).

[24] D. Suzuki, M. Ford, D. Bazin, W. Mittig, et al., Nucl. Inst. Methods in Physics Research A 691, 39 (2012).

[25] http://lise.nscl.msu.edu/lise.html 